\title{
Avaliação da qualidade do sono e sonolência excessiva diurna no Acidente Vascular Cerebral
}

\author{
Assessment of sleep quality and excessive daytime \\ sleepiness in stroke
}

\begin{abstract}
Cintia Raquel de Lima1 ${ }^{1}$, Leila Araújo de Medeiros ${ }^{1}$, Patrícia Cavalcanti da Rocha Leão ${ }^{2}$, Catharinne Angélica Carvalho de Farias², Adriana Jussara Oliveira Brandão²
\end{abstract}

1.Discente de Fisioterapia, Centro Universitário do Rio Grande do Norte, UNI-RN, Natal-RN, Brasil.

2.Fisioterapeuta, Mestre, Centro Universitário do Rio Grande do Norte, UNI-RN, Natal-RN, Brasil.

\begin{abstract}
Resumo
Objetivo. O objetivo do estudo foi avaliar a qualidade de sono e sonolência diurna nas fases aguda e crônica do acidente vascular cerebral (AVC). Método. O estudo foi do tipo transversal e descritivo com uma população constituída por 21 pacientes com sequelas de AVC isquêmico. Os instrumentos utilizados foram: Ficha de Identificação para registro dos dados pessoais e sócio-demográficos, National Institute of Health Stroke Scale, Questionário do Índice de Qualidade de Sono de Pittsburgh e o Questionário de Sonolência de Epworth. Resultados. Os resultados demonstraram não haver correlação entre o comprometimento neurológico, a qualidade de sono e a presença de sonolência excessiva diurna com as fases aguda e crônica, sendo encontrado correlação entre a qualidade do sono e a idade dos pacientes. Conclusões. Portanto, é importante que os profissionais de saúde levem em consideração as possíveis alterações do sono dos pacientes, que possam influenciar seu desempenho funcional.
\end{abstract}

Unitermos. Acidente Vascular Cerebral; Sono; Distúrbios do sono

\begin{abstract}
Objective. Thus, the aim of this study was to assess sleep quality and excessive daytime sleepiness in the acute and chronic phases of the stroke. Method. A cross-sectional and descriptive study was conducted among a population of 21 patients with ischemic stroke. The assessment tools used were: Identification sheet to register socio-demographic and personal data, National Institute of Health Stroke Scale, Pittsburgh Sleep Quality Index (PSQI) questionnaire and Epworth Sleepiness Scale (ESS). Results. The results demonstrated that there is no correlation among neurological impairment, sleep quality and excessive daytime sleepiness with the acute and chronic phases of stroke, however it has been found correlation between sleep quality and patients age. Conclusion. Therefore, it is important that health professionals take into account the possible changes of sleep of patients, which might influence their functional performance.
\end{abstract}

Keywords. Stroke; Sleep; Sleep Disorders

Trabalho realizado no Departamento de Fisioterapia do Centro Universitário do Rio Grande do Norte, UNIRN, Natal-RN, Brasil.

Conflito de interesse: não

Recebido em: 11/08/2015Aceito em: 1/12/2015

Endereço para correspondência: Adriana JO Brandão. Clínica Escola do UNI-RN. R. Prefeita Eliane Barros, 2000. Tirol. CEP 59014-545. Natal-RN, Brasil. E-mail: adrianajbrandao@yahoo.com.br 


\section{INTRODUÇÃO}

Nos últimos anos, o Acidente Vascular Cerebral (AVC) têm sido apontado como uma das principais causas de óbito no Brasil e ocupa o terceiro lugar no cenário mundial. Segundo dados estatísticos do Ministério da Saúde em $2011^{1}$, a taxa de mortalidade para doenças cerebrovasculares no Rio Grande do Sul e no Rio Grande do Norte em 2011 foi respectivamente de 74,4 e 43,3 por 100.000 habitantes ${ }^{2,3}$.

O AVC é uma doença cerebrovascular, definida como um déficit neurológico com permanência dos sintomas acima de 24 horas, e considerada como uma das lesões que mais causa danos permanentes/morbidades para 0 paciente, com consequente incapacidade funcional. Essa patologia pode ser do tipo hemorrágico ou isquêmico, sendo este último 0 tipo mais frequente $(80 \%)$, caracterizado pela oclusão de uma artéria encefálica através de placa de ateroma ou êmbolo, interrompendo o suprimento sanguíneo para determinado área encefálica ${ }^{2,4,5}$.

O AVC isquêmico (AVCI) é um episódio imprevisto que acarreta um grande esforço para uma readaptação após a lesão, visto que surgem novos desafios a serem enfrentados pela existência de alterações físicas, sociais e psicológicas. Dentre as sequelas ocasionadas pelo AVC estão os distúrbios do sono, como por exemplo, a apneia do sono e sonolência excessiva diurna, as quais podem modificar a qualidade de sono e, consequentemente, 
influenciar no resultado do tratamento de fisioterapia. Já, que a qualidade de sono ruim e a sonolência excessiva durante $\mathrm{o}$ atendimento podem retardar $\mathrm{o}$ alcance dos objetivos, devido ao efeito deletério que esses distúrbios têm sobre o estado de alerta e colaboração do paciente ${ }^{6-10}$.

Sendo assim, devido à possibilidade de ocorrência desses distúrbios nos pacientes pós-AVC, o atual estudo visa investigar a qualidade de sono e sonolência excessiva diurna de pacientes com AVCI, comparando a fase aguda com a fase crônica, para observar se há diferença no sono desses dois estágios de recuperação do evento isquêmico.

\section{MÉTODO}

\section{Amostra}

O estudo foi do tipo transversal e analítico com a população constituída por pacientes com sequelas de AVC registrados em Serviços de Saúde na cidade de Natal-RN.

Para composição da amostra, os participantes foram selecionados através do método de amostragem por conveniência, de forma não probabilística e do tipo voluntária, sendo constituída por 21 sujeitos.

Os critérios de inclusão adotados para os pacientes foram: diagnóstico de AVCI unilateral e não recorrente; não possuir transtornos cognitivos graves e afasia; ser alfabetizado; aceitar participar voluntariamente da pesquisa; e possuir idade superior a 40 anos. E os critérios de exclusão foram: não assinar o TCLE e desistir de 
responder aos questionários durante a aplicação. A fase aguda foi considerada como sendo o período até seis meses após o evento isquêmico e a fase crônica a partir dos seis meses após a lesão encefálica ${ }^{11,12}$.

O presente projeto foi aprovado pelo Comitê de Ética em Pesquisa da Liga Norte Riograndense Contra o Câncer (LNRCC) sob o número 073/073/2010.

\section{Procedimentos}

Após a aprovação do projeto foi dado início à coleta de dados. Os participantes foram informados sobre os procedimentos da pesquisa e solicitados a assinar um Termo de Consentimento Livre e Esclarecido (TCLE).

Em seguida, foi aplicada uma Ficha de Identificação para registro dos dados pessoais e sócio-demográficos e a Escala NIHSS (National Institute of Health Stroke Scale) que é um instrumento que possui sua validade e confiabilidade documentada tanto na literatura nacional quanto internacional. Esta escala avalia de forma quantitativa o grau de comprometimento neurológico dos pacientes após um AVC, através de 11 itens que compreendem: nível de consciência, movimentos oculares, campo visual, movimentos faciais, função motora e ataxia de membros superiores e inferiores, assim como sensibilidade, linguagem, presença de disartria e de negligência espacial. Quanto mais elevado o escore do paciente, mais grave é o estado neurológico. O escore total varia de 0 a 42 pontos. Pontuações abaixo de cinco indica 
um nível leve de comprometimento neurológico; valores finais entre 6-13 pontos representam nível moderado de acometimento; e escores finais iguais ou superiores a 14 pontos apontam para um nível de severidade clínica mais avançada $^{13,14}$.

Posteriormente, foi utilizada uma avaliação subjetiva do sono através do Índice de Qualidade de Sono de Pittsburgh (IQSP) e a Escala de Sonolência de Epworth (ESE). O Índice de Qualidade de Sono de Pittsburgh (IQSP) é um questionário que avalia a qualidade subjetiva do sono referente ao mês anterior. Possui sete componentes: latência, duração, eficiência, distúrbios do sono, uso de medicação para dormir e disfunção durante o dia (sonolência diurna). Cada componente varia de 0 a 3, e os escores são somados com valor final variando de 0 a 21 pontos, sendo que escores finais acima de 5 indicam qualidade de sono ruim e escores abaixo ou iguais a 5 indicam qualidade de sono boa?.

A ESE é um questionário que avalia a probabilidade de um indivíduo adormecer em oito situações de atividades diárias, tais como sentado e lendo; assistindo TV; sentado, quieto, em um lugar público (por exemplo, em um teatro, reunião ou palestra); andando de carro por uma hora sem parar, como passageiro; sentado quieto após o almoço sem bebida de álcool; em um carro parado no trânsito por alguns minutos. O escore final varia de 0 a 24, de modo que os escores acima de 10 sugerem sonolência diurna 
excessiva (SDE) e escores menores ou iguais a 10 representam ausência de $\mathrm{SDE}^{15}$.

\section{Análise Estatística}

A análise dos dados foi feita de forma descritiva para caracterizar o perfil epidemiológico da amostra e para demais dados foi realizada através do programa SPSS 22.0 (Statistical Package for the Social Science), atribuindo-se o nível de significância menor do que 5\% para todos os testes estatísticos.

Para analisar a normalidade dos dados foi utilizado o teste de Shapiro-Wilk, visto que a amostra foi composta por 21 sujeitos. A associação entre o grau de comprometimento neurológico dos pacientes pós-AVC, nível de sonolência diurna e qualidade do sono com as fases aguda ou crônica foi feita através do teste qui-quadrado de Pearson, e para comparar a idade dos sujeitos com a qualidade do sono utilizou-se o teste de Mann-Whitney para amostras independentes.

\section{RESULTADOS}

A amostra investigada foi caracterizada quanto aos aspectos pessoais (gênero, estado civil, idade e escolaridade) e aspectos clínicos (hemisfério acometido, tempo de sequela e comorbidades). Os resultados encontrados mostraram uma amostra de 21 indivíduos, predominantemente do gênero feminino $(71,4 \%)$, faixa 


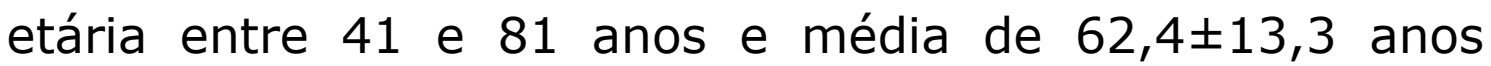

(Figura 1). O nível de escolaridade predominante foi 0 Ensino Fundamental (71,4\%) e estado civil casado $(66,7 \%)$, seguido de viúvo $(19,0 \%)$, como apresentados na Tabela 1.

Figura 1. Distribuição da faixa etária.
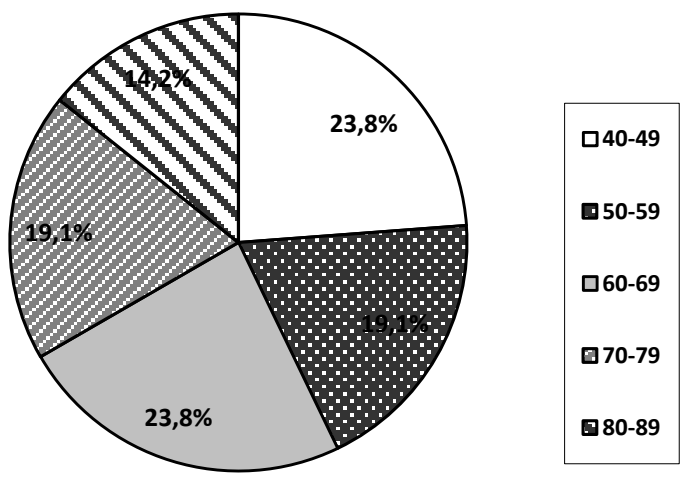

Tabela 1. Caracterização sociodemográfica e clínica nos 21 pacientes.

\begin{tabular}{ll}
\hline Variáveis & $\%(\mathrm{n})$ \\
\hline Gênero & \\
$\quad$ Feminino & $71,4(15)$ \\
Masculino & $28,6(6)$ \\
Estado Civil & $66,7(14)$ \\
$\quad$ Casado & $9,5(2)$ \\
Solteiro & $19,0(4)$ \\
Viúvo & $4,8(1)$ \\
$\quad$ Divorciado & \\
Escolaridade & $9,5(2)$ \\
Alfabetização & $61,9(13)$ \\
Ensino Fundamental & $28,6(6)$ \\
Ensino Médio & \\
Tempo de Sequela & $52,3(11)$ \\
$\quad$ Aguda & $47,7(10)$ \\
$\quad$ Crônica & \\
Hemisfério acometido & $57,2(12)$ \\
$\quad$ Esquerdo & $42,8(9)$ \\
Direito & \\
Comorbidades Clínicas & \\
$\quad$ Hipertensão Arterial Sistêmica & $85,7(18)$ \\
Diabetes Mellitus & $47,6(10)$ \\
$\quad$ Tabagismo & $4,8(1)$ \\
\hline
\end{tabular}


Com relação às características clínicas, o tempo de sequela após o AVCI foi predominantemente classificado como agudo $(52,3 \%)$, o hemisfério mais acometido foi o esquerdo $(57,2 \%)$ e dentre as comorbidades clínicas questionadas a mais dominante foi a Hipertensão Arterial Sistêmica $(85,7 \%)$, seguida da Diabetes Mellitus $(47,6 \%)$ como descritas na Tabela 1.

O grau de comprometimento neurológico avaliado através da escala NIHSS observou que 52,4\% da amostra tiveram um comprometimento neurológico leve, 47,6\% moderado e nenhum indivíduo apresentou comprometimento neurológico grave. A Sonolência Excessiva Diurna (SED) investigada pela ESE estava presente em apenas $19 \%$ da amostra. A qualidade subjetiva do sono avaliada por meio do IQSP foi caracterizada como boa em 71,4\% dos indivíduos (Tabela 2).

Tabela 2. Distribuição (em porcentagem) dos resultados dos questionários de acordo com a fase do AVC.

\begin{tabular}{lcc}
\hline \multicolumn{1}{c}{ Fase do AVC } & Agudo & Crônico \\
$\begin{array}{l}\text { Vampróveis } \\
\text { Neurológico }\end{array}$ & & \\
$\quad$ Leve & 33,3 & \\
$\quad$ Moderado & 19,1 & 19,1 \\
Sonolência Excessiva Diurna & & 28,5 \\
$\quad$ Sim & 14,3 & 4,8 \\
Não & 38,1 & 42,8 \\
Qualidade Subjetiva do Sono & & \\
$\quad$ Boa & 38,1 & 33,3 \\
$\quad$ Ruim & 14,3 & 14,3 \\
\hline
\end{tabular}


Ao analisar possíveis correlações entre a fase do AVC (aguda e crônica) e as variáveis estudadas nos questionários, observou-se não haver correlação entre elas, como visto na Tabela 3. Evidenciando, portanto, que a fase do AVC não influenciou nas variáveis citadas, para a amostra estudada.

Tabela 3. Correlação do grau de comprometimento neurológico, qualidade do sono e sonolência excessiva diurna com as fases do AVC.

\begin{tabular}{lcc}
\hline $\begin{array}{l}\text { Instrumentos de } \\
\text { Avaliação }\end{array}$ & R & $\begin{array}{c}\text { p- } \\
\text { valor }\end{array}$ \\
\hline NIHSS & 0,12 & 0,249 \\
IQSP & 0,89 & 0,890 \\
ESE & 0,30 & 0,652
\end{tabular}

No entanto, entre a idade e a qualidade do sono, encontrou-se correlação demonstrando que os pacientes com faixa etária mais elevada tendem a ter uma pior qualidade de sono $(p=0,02 ;$ Figura 2$)$.

Figura 2. Correlação da qualidade de sono com a idade dos pacientes.

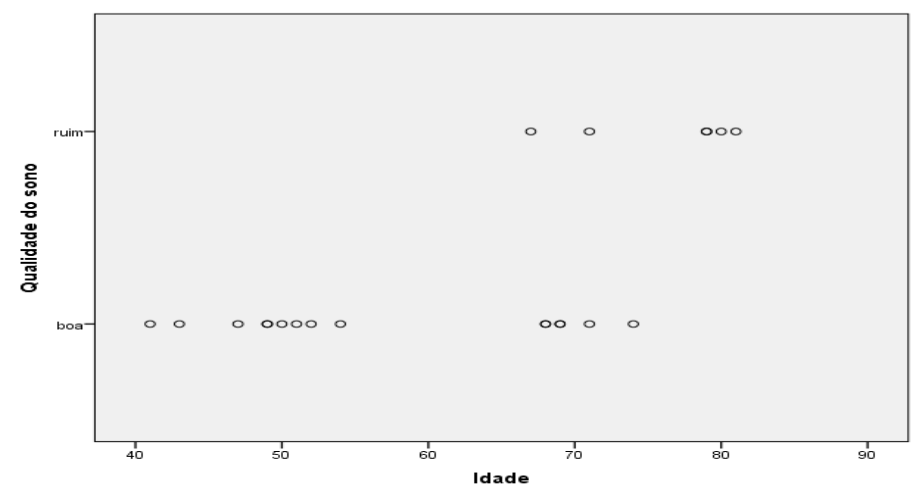




\section{DISCUSSÃO}

As características sociodemográficas da amostra evidenciaram que a incidência de AVCI apresenta-se principalmente após os 60 anos, corroborando com os achados da literatura que mostram um aumento gradual com o avançar da idade ${ }^{16,17}$.

Estudos realizados demonstram que essa população constitui-se principalmente do gênero feminino, estado civil com maior predominância sendo o casado seguido de viúvo ${ }^{13}$ e como grau de escolaridade o ensino fundamental ${ }^{14,18}$, concordando com os achados do presente estudo. No entanto, discordando da presente pesquisa, em um estudo realizado no Nordeste com 962 indivíduos, o maior percentual da amostra $(52,3 \%)$ foi do sexo masculino ${ }^{19}$.

O hemisfério cerebral mais acometido corrobora com os achados da maioria dos estudos que demostram que o hemisfério esquerdo é mais vitimado ${ }^{13}$. Dentre as comorbidades que geralmente precedem ou cursam com o evento isquêmico, a literatura aponta em maior percentual a Hipertensão Arterial Sistêmica ${ }^{19}$, seguida de Diabetes Mellitus e Tabagismo apresentando a mesma ordem na amostra que foi avaliada ${ }^{20}$.

Um estudo realizado com 40 pacientes na fase aguda do AVC na cidade de Natal observou que houve a presença de severidade clinica (NIHSS) classificada como moderada $(57,5 \%)$ seguida de severa $(42,5 \%)^{14}$, discordando deste estudo já que não houve a presença de comprometimento 
grave e a fase aguda apresentou comprometimento neurológico leve, seguido de moderado. No entanto outro estudo que avaliou o grau neurológico encontrou de acordo com o NIHSS, comprometimento neurológico leve (77\%) seguido de moderado (23\%) em uma amostra com 12 indivíduos, corroborando com os nossos achados que apresentou 52,4\% da amostra classificada como comprometimento neurológico leve ${ }^{21}$.

Não foi encontrada a presença de correlação da fase do AVC com o comprometimento neurológico, qualidade de sono e sonolência excessiva diurna. Talvez em função do tamanho reduzido da amostra, já que era esperado que houvesse diferença entre as fases.

De acordo com a literatura, o AVC está entre as causas médicas que podem levar à presença de sonolência excessiva diurna ${ }^{22}$. Em um estudo realizado na fase aguda do AVCI constatou-se a presença de sonolência excessiva diurna $(34,8 \%)$ e de qualidade de sono ruim $(65,2 \%)$ nos pacientes avaliados ${ }^{23}$. Já no presente estudo, observou-se a presença de SED em 19,1\% da amostra e qualidade de sono classificada como ruim em $28,6 \%$ dos indivíduos avaliados.

Geralmente, concomitantemente com as várias alterações anátomo-funcionais que ocorrem após o AVC, há eventos de apneia que podem influenciar na qualidade do sono e ocasionar sonolência excessiva diurna. Desta forma, além da avaliação neurológica, a avaliação subjetiva do 
sono se faz necessária, embora muitas vezes seja negligenciada ${ }^{24}$.

Com o avançar da idade há modificações na qualidade do sono do indivíduo. Concordando com os nossos achados, indivíduos com idade mais avançada apresentam uma pontuação mais elevada no IQSP, indicando uma qualidade de sono ruim ${ }^{25-27}$, justificando dessa forma a presença de correlação estatisticamente significativa entre a idade dos sujeitos e a qualidade de sono.

A qualidade de sono ruim em idosos ocasiona a presença de sonolência excessiva diurna ${ }^{25}$, o que provavelmente justifica a presença de sonolência excessiva mesmo em pequena porcentagem no presente estudo.

A presença de sonolência excessiva diurna além de contribuir para uma qualidade de sono ruim compromete atividades físicas e influencia nas atividades laborais do paciente, repercutindo também na reabilitação pós AVC e na qualidade de vida $22,28,29$.

Portanto, é necessário avaliar a qualidade do sono e identificar possíveis alterações que o paciente possa apresentar, para que sejam realizados encaminhamentos e tratamentos necessários aos profissionais apropriados 0 mais precocemente possível.

Neste sentido evidencia-se a necessidade de estudos com um número amostral maior, já que o tamanho da amostra é considerada como limitação deste estudo, correlacionando também o cronotipo do paciente (matutino, 
vespertino ou indiferente) com o horário da fisioterapia já que isso pode influenciar o desempenho da reabilitação pós AVC, seja esta na fisioterapia, fonoaudiologia ou terapia ocupacional, entre outras.

\section{CONCLUSÃO}

No presente estudo os pacientes foram classificados com comprometimento neurológico seguido de grave, observou-se uma discreta presença de sonolência excessiva diurna, principalmente na fase aguda do AVCI e boa qualidade subjetiva do sono nas fases aguda e crônica. A faixa etária superior a 60 anos apresentou uma pior qualidade de sono em relação a idades inferiores.

\section{AGRADECIMENTOS}

Suporte Financeiro do Centro Universitário do Rio Grande do Norte, UNI-RN e do Conselho Nacional de Desenvolvimento Científico e Tecnológico, CNPQ.

Agradecemos as Clínicas Integradas do UNI-RN e ao Centro de Reabilitação Adulto CRA na cidade do Natal por nos permitir acesso aos pacientes em tratamento.

\section{REFERÊNCIAS}

1.Sistema de informações sobre mortalidade. DATASUS (endereço na internet). Brasil: Ministério da Saúde. (última atualização 04/2015; acessado em 09/2014). Disponível em: http://tabnet.datasus.gov.br/ 
2.Polese JC, Tonial A, Jung FK, Mazuco R, Oliveira SG, Schuster RC. Avaliação da funcionalidade de indivíduos acometidos por Acidente Vascular Encefálico. Rev Neurocienc 2008;16:175-8.

3.Leite HR, Nunes APN, Corrêa CL. Perfil epidemiológico e qualidade de vida dos pacientes acometidos por acidente vascular encefálico. Arq Cienc Saúde UNIPAR 2011;15:15-21. http://dx.doi.org/10.1590/S1809-29502009000100007

4.Paulo RB, Guimaraes TM, Helito PVP, Marchiori PE, Yamamoto FI, Mansur LL, et al. Acidente vascular cerebral isquêmico em uma enfermaria de neurologia: complicações e tempo de internação. Rev Assoc Med Bras 2009;55:313-6. http://dx.doi.org/10.1590/S010442302009000300025

5.Barros AFF, Fábio SRC, Furkim AM. Correlação entre os achados clínicos da deglutição e os achados da tomografia computadorizada de crânio em pacientes com acidente vascular cerebral isquêmico na fase aguda da doença. Arq Neuropsiquiatr 2006;64:1009-14. http://dx.doi.org/10.1590/S0004-282X2006000600024

6.Pires SL, Gagliardi RJ, Gorzoni ML. Estudo das freqüências dos principais fatores de risco para acidente vascular cerebral isquêmico em idosos. Arq Neuropsiquiatr 2004;62:844-51.

http://dx.doi.org/10.1590/S0004-282X2004000500020

7.Campos TF, Rodrigues CA, Farias IMA, Ribeiro TS, Melo LP. Comparação dos instrumentos de avaliação do sono, cognição e função no acidente vascular encefálico com a classificação internacional de funcionalidade, incapacidade e saúde (CIF). Rev Bras Fisioter 2012;16:23-9.

http://dx.doi.org/10.1590/S1413-35552012000100005

8.Bittencourt LRA, Silva RS, Santos RF, Pires MLN, Mello MT. Sonolência excessiva. Rev Bras Psiquiatr 2005;27:16-21. http://dx.doi.org/10.1590/S1516-44462005000500004

9.Perlini NMOG, Faro ACM. Cuidar de pessoa incapacitada por acidente vascular cerebral no domicílio: o fazer do cuidador familiar. Rev Esc Enferm USP 2005;39:154-63. http://dx.doi.org/10.1590/S0080-62342005000200005

10.Caneda MAG, Fernandes JG, Almeida AG, Mugnol FE. Confiabilidade de escalas de comprometimento neurológico em pacientes com acidente vascular cerebral. Arq Neuropsiquiatr 2006;64:690-7. http://dx.doi.org/10.1590/S0004-

282X2006000400034

11.Silva MS, Corrêa JCF, Silva FC, Sampaio LMM, Corrêa FI. Comparação da força muscular respiratória entre idosos após acidente vascular cerebral. Acta Fisiatr 2013;20:20-3. https://doi.org/10.5935/0104-7795.20130004

12.Scianni A, Salmela LFT, Ada L. Challenges in recruitment, attendance and adherence of acute stroke survivors to a randomized trial in Brazil: a feasibility study. Rev Bras Fisioter 2012;16:40-5.

http://dx.doi.org/10.1590/S1413-35552012000100008

13. Costa FA, Silva DLA, Rocha VM. Estado neurológico e cognição de pacientes pós-acidente vascular cerebral. Rev Esc Enferm USP 
62342011000500008

14.Costa FA, Silva DLA, Rocha VM. Severidade clínica e funcionalidade de pacientes hemiplégicos pós-AVC agudo atendidos nos serviços públicos de fisioterapia de Natal. Cienc Saúde Colet 2011;16:1341-8.

http://dx.doi.org/10.1590/S1413-

$\underline{81232011000700068}$

15.Bertolazi AN, Pagondes SC, Hoff LS, Pedro VD, Barreto SM, Johns MW. Validação da escala de sonolência de Epworth em português para uso no Brasil. J Bras Pneumol 2009;35:877-83.

http://dx.doi.org/10.1590/S1806-37132009000900009

16.Apolinário ES, Santos EFS, Carneiro JNP, Fernandes CDM, Souza RAS. Kinesio Taping como auxiliar na recuperação de pacientes pósAVC no membro superior par ético: um ensaio clínico. Rev Inter Saúde Hum Tec 2014;2:1-4. http://dx.doi.org/10.16891/149

17.Lopes JM, Medeiros JLA, Oliveira KBA, Dantas FG. Acidente vascular cerebral isquêmico no Nordeste brasileiro: uma análise temporal de 13 anos de casos de hospitalização. ConScient Saúde 2013;12:321-8. https://doi.org/10.5585/conssaude.v12n2.4100

18. Dantas AATSG, Torres SVS, Farias IMA, Sant'Ana SBCL, Campos TF. Rastreio cognitivo em pacientes com acidente vascular cerebral: um estudo transversal. J Bras Psiquiatr 2014;63:98-103.

http://dx.doi.org/10.1590/0047-2085000000012

19.Fernandes TG, Goulart AC, Campos TF, Lucena NMG, Freitas KLA, Trevisan CM, et al. Early stroke case-fatality rates in three hospital registries in the Northeast and Southeast of Brazil. Arq Neuropsiquiatr 2012;70:869-73.

http://dx.doi.org/10.1590/S0004-

282X2012001100009

20.Rodrigues ESR, Castro KAB, Rezende AAB, Herrera SDSC, Pereira AM, Takada JAP. Fatores de risco cardiovascular em pacientes com acidente vascular cerebral. Rev Amazon 2013;1:21-8.

21.Campos TF, Dantas AATSG, Melo LP, Oliveira DC. Grau neurológico e funcionalidade de pacientes crônicos com acidente vascular cerebral: Implicações para a prática clínica. Arq Cinc Saúde 2014;21:28-33.

22.Giorelli AS, Santos PP, Carnaval T, Gomes MMG. Sonolência excessiva diurna: aspectos clínicos, diagnósticos e terapêuticos. Rev Bras Neurol 2012;48:17-24.

23. Medeiros CAM, Bruin MS, Silva CC, Araújo SMHA, Júnior Chaves $\mathrm{CM}$, Bruin PFC. Neck circumference, a bedside clinical feature related to mortality of acute ischemic stroke. Rev Assoc Med Bras 2011;57:559-64. http://dx.doi.org/10.1590/s0104-

42302011000500015

24.Cintra FD, Poyares D, Guilleminault C, Carvalho AC, Tufik S, Paola AAV. Alterações Cardiovasculares na Síndrome da Apnéia Obstrução do Sono. Arq Bras Cardiol 2006;86:399-407. http://dx.doi.org/10.1590/S0066-782X2006000600001 
25.Quinhones MS, Gomes MM. Sono no envelhecimento normal e patológico: aspectos clínicos e fisiopatológicos. Rev Bras Neurol 2011;47:31-42.

26.Araújo CLO, Ceolim MF. Qualidade do sono de idosos residentes em instituição de longa permanência. Rev Esc Enferm USP 2010;44:619-26.

http://dx.doi.org/10.1590/S0080-62342010000300010

27.Corrêa K, Ceolim MF. Qualidade do sono em pacientes idosos com patologias vasculares periféricas. Rev Esc Enferm USP 2006;42:12-8. http://dx.doi.org/10.1590/S0080-62342008000100002

28. Marqueze EC, Silva MJ, Moreno CRC. Qualidade de sono, atividade física durante o tempo de lazer e esforço físico no trabalho entre trabalhadores noturnos de uma indústria de cerâmica. Rev Bras Saúde Ocup 2009;34:93-100.

http://dx.doi.org/10.1590/S0303-76572009000100011

29.Ambrósio P, Geib LTC. Sonolência excessiva diurna em condutores de ambulância da Macrorregião Norte do Estado do Rio Grande do Sul, Brasil. Epidemiol Serv Saúde 2008;17:21-31. http://dx.doi.org/10.5123/S1679-49742008000100003 